\title{
Reference Role of Middle Turbinate Axilla in Lacrimal Sac Localization Assisted by Computed Tomographic Dacryocystography
}

\section{Lihong Yang ( $\nabla$ ylhong922@126.com )}

Tianjin Eye Hospital, Affiliated Eye Hospital of NanKai University, Clinical College of Ophthalmology of Tianjin Medical University

Hongxun Li

Tianjin Eye Hospital, Affiliated Eye Hospital of NanKai University, Clinical College of Ophthalmology of Tianjin Medical University

\section{Zhi Yin}

Tianjin Eye Hospital, Affiliated Eye Hospital of NanKai University, Clinical College of Ophthalmology of Tianjin Medical University

\section{Lei Zhang}

Tianjin Eye Hospital, Affiliated Eye Hospital of NanKai University, Clinical College of Ophthalmology of Tianjin Medical University

\section{Zhenhai Yang}

Tianjin Eye Hospital, Affiliated Eye Hospital of NanKai University, Clinical College of Ophthalmology of Tianjin Medical University

\section{Research Article}

Keywords: lacrimal sac, middle turbinate axilla, CT-DCG, common canaliculus, SSF

Posted Date: September 22nd, 2021

DOl: https://doi.org/10.21203/rs.3.rs-917275/v1

License: (c) (1) This work is licensed under a Creative Commons Attribution 4.0 International License. Read Full License 


\section{Abstract}

To investigate the influencing factors and availability of locating the lacrimal sac by the reference position of the middle turbinate axilla (MTA) on computed tomographic dacryocystography (CT-DCG) images. A series of 192 endoscopic dacryocystorhinostomy (DCR) surgeries were performed in 162 patients . All the patients had been diagnosed as nasolacrimal duct obstruction and received CT-DCG examinations. According to the maximum transverse diameter of the lacrimal sac on CT-DCG, the patients were classified into three groups. Measurements were taken on CT-DCG parasagittal images. The average distance from the sac superior fundus (SSF) to the MTA was $7.52 \mathrm{~mm} \pm 3.23 \mathrm{~mm}$, and it increased with the increase of the maximum transverse diameter of sac among groups $(p<0.01)$. The average distance from the common canaliculus (CC) to the MTA was $3.95 \mathrm{~mm} \pm 2.49 \mathrm{~mm}$. No significant difference among the groups $(p=0.11)$. The average distance from the CC to the SSF was $3.41 \mathrm{~mm} \pm 1.31 \mathrm{~mm}$, and it increased with the increase of the sac transverse diameter among groups $(p<0.01)$. The lacrimal sac can be accurately located on the lateral nasal wall by the reference position of the MTA and the CC on CT-DCG images. The distance of the SSF to the MTA was influenced by the lacrimal sac size. The distance of the CC to the MTA was more constant on CT-DCG images.

\section{Introduction}

Endoscopic dacryocystorhinostomy (DCR) surgery is an effective treatment for managing nasolacrimal duct obstruction and dacryocystitis by reconstructing the lacrimal drainage system (LDS) of nasal ${ }^{1}$. In previous studies, the high success rate of endoscopic DCR ranged from 80-95\%. However, there was still $4-13 \%$ surgical failure occurred ${ }^{2-3}$. The main reasons for failure were associated with inappropriate position of the ostium, extra damage of mucosa around the lacrimal sac and inadequate exposure of the lacrimal $\mathrm{sac}^{4}$. Accurate positioning of the lacrimal sac are contributing to the formation of ideal DCR ostium, which is the key to improving the success rate of DCR and achieving effective lacrimal drainage.

Computed tomographic dacryocystography (CT-DCG) can be used to locate the lacrimal sac, and it is proven to be a safe, objective, non-invasive and reliable diagnostic method for assessing the nasolacrimal duct system in patients prior to DCR. The sac superior fundus (SSF) and the common canaliculus (CC) are the main signs of lacrimal sac on CT-DCG. The middle turbinate axilla (MTA) has always been used as a stable anatomic landmark for endoscopic surgeons to locate the lacrimal sac on the lateral nasal wall. The position of the lacrimal sac is commonly found to be anterior to the MTA, and two-thirds of the lacrimal sac length are superior to the insertion of the middle turbinate on the lateral nasal wall ${ }^{5}$. In previous CT-DCG study, the SSF is shown extending $8-10 \mathrm{~mm}$ above the the middle turbinate insertion, and the height of the sac above the common canalicular opening is approximately $5 \mathrm{~mm}^{6}$. However, does this sac location theory apply to everyone? For different size of the sac, does the distance of the SSF to the MTA remain stable? How about the distance of the CC to the MTA? Theses problems had rarely been discussed in earlier studies. In this retrospective study, the average distance from the SSF and the CC to the MTA was studied on CT-DCG Images in groups, aiming to investigate the 
influencing factor (lacrimal sac size) and availability of locating the lacrimal sac by the reference position of the middle turbinate axilla. To our knowledge, there have been few related study reported before.

\section{Materials And Methods}

The retrospective study was performed in accordance with the declaration requirement of Helsinki for research involving human subjects. The protocol and waiver of informed consent used in this study were approved by the Ethics Board of Tianjin Eye Hospital.

Consecutive endoscopic DCR patients from January 2018 to December 2020 were included in the study. Medical records including clinical information and surgical notes and videos were reviewed. A total of 192 eyes on 162 patients were included in the study and underwent CT-DCG and subsequent endoscopic DCRs. Lacrimal irrigation was used as a standard preoperative evaluation on the patency and reflux of lacrimal drainage system. Nasal endoscopic exam was used to determined the necessity of additional nasal surgery (septoplasty, middle turbinoplasty or sinus surgery) preoperatively. All the Patients included in the study had been diagnosed as nasolacrimal duct obstruction. The exclusion criteria were history of facial bone fracture, previous lacrimal surgery, obstruction of lacrimal canaliculus, lacrimal canaliculi inflammation and children. Patients who had acute dacryocystitis process and suspected lacrimal drainage tumor were also excluded. Patients who underwent additional nasal surgery were included in the analysis.

In order to discharge purulent secretion, all the patients underwent lacrimal irrigation with saline before performing DCG. And 0.5-1.0ml of water-soluble contrast medium (lohexol, $755 \mathrm{mg} / \mathrm{ml}$ ) was slowly injected into the examined LDS from inferior as well as superior punctum. Axial view CT scan with a thickness of $1 \mathrm{~mm}$ was performed with soft-tissue and bone windows. And axial images data were reconstructed into coronal and parasagittal images along the main axis of the LDS for subsequent measurements. Measurements on the CT images were performed using software on a GE Advantage Windows workstation. All DCG images were evaluated by two experienced adiologists, and the final agreement was reached by consensus.

According to the maximum transverse diameter of the lacrimal sac on CT-DCG, the patients were classified into three groups. 1) large lacrimal sac (LLS), the maximum sac transverse diameter $>5 \mathrm{~mm}$; 2)medium lacrimal sac (MLS), the maximum sac transverse diameter was2-5mm; 3)small lacrimal sac (SLS), the maximum sac transverse diameter $<2 \mathrm{~mm}$.

All measurements below were taken from the long axis of the lacrimal sac in three groups respectively (see Fig. 1). According to Wormald measuring method ${ }^{5}$, a line was drawn from the MTA to the long axis of the sac at right angles, which was used to measure the height of the sac above the middle turbinate. Another line was drawn through the $\mathrm{CC}$ at right angles to the sac long axis, which was used to measure the height of sac above the $\mathrm{CC}$. And the distance from the MTA to the $\mathrm{CC}$ was calculated by the difference 
between the above two height. Meanwhile, the thickness of the maxillary frontal process (MFP) was measured on the line drawn through the CC.

According to the reference position of the middle turbinate insertion on CT-DCG, the plane of the CC and that of the SSF were marked on the lateral nasal wall with graduated probe. Furthermore, the surgical incision of nasal mucosa was designed. All patients were performed with standardized endoscopic DCR technique which was described by Wormald previously. ${ }^{7}$ The SSF and the opening of the CC were exposed and intraoperative measurements were performed (see Fig. 2). Surgical outcome was not the focus of discussion, so there was no further success rate analysis in this study.

\section{Statistical Analysis}

The results of continuous variables were presented as mean \pm standard deviation (SD), whereas that of categorical variables were presented as percentages. Differences of study groups were compared in terms of mean age, the distance between the SSF, CC and the MTA using the one-way analysis of variance (ANOVA). LSD Post hoc test was used for multiple comparison after it. Chi-square or Fisher's exact test was used to compare proportions of gender, side, poor imaging LDSs eyes, and combined additional nasal surgery. Data were analyzed with IBM SPSS 24 statistical software. All tests were twosided, and $p<0.05$ was considered as statistically significant.

\section{Result}

One hundred and sixty two consecutive patients, 125women and 37 men, mean age 52.8years (range2283), were recruited to the study. Of all patients, there were 30 patients with binocular diseases and 132 patients with monocular diseases. CT-DCG was performed on affected eyes in patients. No CT-DCG related side effects occurred. All lacrimal sac images can be detected on CT-DCG. Nevertheless, the images of lacrimal canaliculi or CC in 12 eyes (8 patients) were not clearly displayed and were excluded. In 53 eyes (43patients), the images of the canaliculi or CC that were thin but did not affect subsequent image measurements were included in the analysis. Demographic data and preoperative findings are summarized in Table 1. All patients underwent endoscopic DCRs, and 17 DCRs (17 patients) underwent endoscopic DCR combined with additional nasal surgery (see Table 2). There was no statistically significant difference in mean age, gender, side, poor imaging LDSs cases, combined with additional nasal surgery between study groups. 
Table 1

Comparison of groups' demographic data and preoperative findings

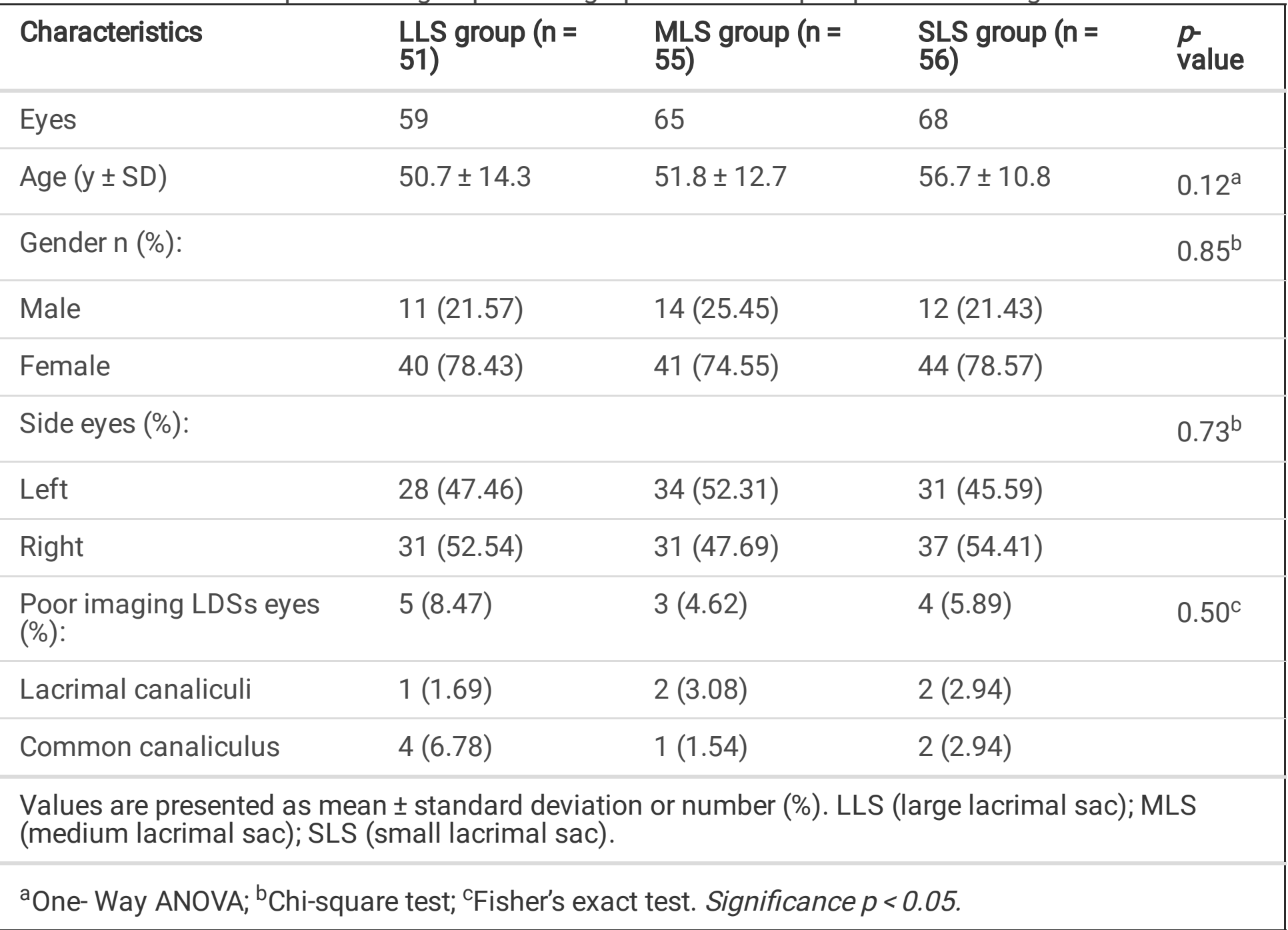

Table 2

The distribution of primary dacryocystorhinostomy combined with additional nasal surgery in the study groups

\begin{tabular}{|c|c|c|c|c|}
\hline Characteristics & $\begin{array}{l}\text { LLS group } \\
(\text { eye }=54)\end{array}$ & $\begin{array}{l}\text { MLS group } \\
(\text { eye }=62)\end{array}$ & $\begin{array}{l}\text { SLS group } \\
(\text { eye }=64)\end{array}$ & $\begin{array}{l}p- \\
\text { value }\end{array}$ \\
\hline $\begin{array}{l}\text { Primary DCR + additional nasal } \\
\text { surgery eyes }(\%) \text { : }\end{array}$ & $5(9.26)$ & 7 (11.29) & $5(7.81)$ & $0.99^{a}$ \\
\hline Septoplasty & $1(1.85)$ & $2(3.23)$ & $2(3.13)$ & \\
\hline $\begin{array}{l}\text { Middle } \\
\text { turbinoplasty }\end{array}$ & $3(5.56)$ & $4(6.45)$ & $2(3.13)$ & \\
\hline Sinus surgery & $1(1.85)$ & $1(1.61)$ & $1(1.56)$ & \\
\hline
\end{tabular}


The surgical data showed that the location of lacrimal sac during the operation was basically consistent with the measurements of CT-DCG lacrimal sac positioning. The measurements between the SSF, the CC and the MTA in the long axis of the sac were presented in Table 3.

Table 3

Measurements of computed tomographic dacryocystography (CT-DCG) images in study groups

\begin{tabular}{|c|c|c|c|c|}
\hline $\begin{array}{l}\text { Measurements on CT-DCG } \\
\text { images }(\mathrm{mm})\end{array}$ & $\begin{array}{l}\text { LLS group (eye } \\
=54 \text { ) }\end{array}$ & $\begin{array}{l}\text { MLS group (eye } \\
=62 \text { ) }\end{array}$ & $\begin{array}{l}\text { SLS group (eye } \\
=64)\end{array}$ & $\begin{array}{l}p- \\
\text { value }\end{array}$ \\
\hline MTA - SSF & $8.63 \pm 3.05$ & $7.59 \pm 2.92$ & $5.96 \pm 2.56$ & $0.00^{\mathrm{a}}$ \\
\hline MTA - CC & $4.25 \pm 2.76$ & $4.12 \pm 2.63$ & $3.33 \pm 1.79$ & $0.14^{\mathrm{a}}$ \\
\hline CC - SSF & $4.38 \pm 1.05$ & $3.46 \pm 1.20$ & $2.63 \pm 1.15$ & $0.00^{\mathrm{a}}$ \\
\hline $\begin{array}{l}\text { Thickness of MFP at the level of } \\
\text { CC }\end{array}$ & $3.47 \pm 1.27$ & $3.49 \pm 1.28$ & $3.56 \pm 1.13$ & $0.94^{\mathrm{a}}$ \\
\hline \multicolumn{5}{|c|}{$\begin{array}{l}\text { Values are presented as mean } \pm \text { standard deviation. CT-DCG (computed tomographic } \\
\text { dacryocystography); LLS (large lacrimal sac); MLS (medium lacrimal sac); SLS (small lacrimal sac); } \\
\text { MTA (middle turbinate axilla); SSF (sac superior fundus); CC (common canaliculus); MFP (maxillary } \\
\text { frontal process). }\end{array}$} \\
\hline \multicolumn{5}{|c|}{${ }^{\mathrm{a} O n e-}$ Way ANOVA; Significance $p<0.05$. } \\
\hline
\end{tabular}

Of all patients, the SSF was located above the MTA on CT-DCG images, and the average distance from the SSF to the MTA was $7.52 \mathrm{~mm} \pm 3.23 \mathrm{~mm}$. According to the aforementioned grouping, the average distance from the SSF to the MTA increased with the increase of the maximum transverse diameter of sac in groups. There was a significant difference between groups $(p<0.01)$ (see Fig. $3 A$ ).

Of all 180 eyes, the CC of 6 eyes (3.33\%) were located below the MTA, and that of 174 eyes (96.7\%) were above. The average distance from the CC to the MTA was $3.95 \mathrm{~mm} \pm 2.49 \mathrm{~mm}$. There was no significant difference among the groups $(p=0.11)$ (see Fig. 3B).

In all cases, the average distance from the CC to the SSF was $3.41 \mathrm{~mm} \pm 1.31 \mathrm{~mm}$. Moreover, the average distance from the CC to the SSF increased with the increase of the maximum transverse diameter of sac in groups. The difference among groups was statistically significant $(p<0.01)$ (see Fig. 3C).

The thickness of the MFP at the level of the CC was measured, and the average thickness was 3.50mm \pm $1.24 \mathrm{~mm}$. There was no significant difference between groups $(p=0.70)$ (see Fig. 3D).

\section{Discussion}

Most of the reasons for the failure of endoscopic DCR can be attributed to a poor understanding of endonasal anatomy and the lacrimal sac position on the lateral nasal wall, which can lead to a wrong location of the rhinostomy ${ }^{5}$. Different from the procedure of external approach, the first step of endonasal 
DCR is to determine the projection position of the lacrimal sac on the lateral nasal wall. In order to locate the lacrimal sac accurately, it is necessary to find a stable surgical reference mark and determine its relative position with lacrimal sac on the lateral nasal wall.

The axilla of the middle turbinate is a constant endonasal anatomical landmark, which can be utilized to localize the lacrimal sac in DCR surgery. Wormald et al. 's study on CT-DCG showed that the sac is located 8-10 $\mathrm{mm}$ above the axilla of the middle turbinate ${ }^{6}$. In other studies, the fundus of the sac is an average of $4.73 \mathrm{~mm} \pm 2.86 \mathrm{~mm}, 6.6 \mathrm{~mm} \pm 1.3 \mathrm{~mm}$ above the axilla of the middle turbinate, respectively ${ }^{8-9}$. Apart from the ethnic origin, were the differences in these findings influenced by other factors, such as size? Previous studies have rarely addressed the above issues further.

Through the measurement of CT-DCG images, SSF of all cases was located above the MTA in this study. And the average distance from the axilla to the sac fundus was $7.52 \mathrm{~mm} \pm 3.23 \mathrm{~mm}$. Our result is very close to the results of Wormald et al. However, this study grouped according to the transverse diameter of the lacrimal sac showed that the average distance from the axilla to the sac fundus in the large, medium, and small lacrimal sac groups was $8.63 \mathrm{~mm} \pm 3.05 \mathrm{~mm}, 7.59 \mathrm{~mm} \pm 2.92 \mathrm{~mm}, 5.96 \mathrm{~mm} \pm 2.56 \mathrm{~mm}$, respectively. With the increase of the transverse diameter of the sac, the distance from the the axilla to the sac fundus gradually increased. Our results show that the size of the lacrimal sac is an important factor affecting the relative position of the superior fundus of the sac. The relative position of the lacrimal sac fundus is not constant.

In our study, the CC of most patients is above the MTA, and the average distance from the CC to the axilla is $3.95 \mathrm{~mm} \pm 2.49 \mathrm{~mm}$. Moreover, there was no statistically significant difference between groups. These results indicate that the position of the $\mathrm{CC}$ relative to the axilla is relatively stable and is less affected by the size of the lacrimal sac. Because the $\mathrm{CC}$ is interwoven and surrounded by hard and thick tissues such as muscle fibers and medial canthal ligaments, it is speculated that the relatively stable position of the $\mathrm{CC}$ may be related to these anatomical factors ${ }^{10}$. One of the main reasons given for the failure of external DCR surgery is inadequate bone removal in the sac projection area, which may also be an important factor for the failure of endoscopic DCR surgery ${ }^{5}$. The CC provides a valuable landmark for endoscopic surgeons. If the $\mathrm{CC}$ is visible through the open sac, the surgeon can be reassured that the bone removal are sufficiently high and most of the sac is exposed. In ideal DCR procedure, the area of bony resection around the $\mathrm{CC}$ should be at least $3-5 \mathrm{~mm}$ in diameter ${ }^{6,11}$. If the level of the $\mathrm{CC}$ is determined on the lateral nasal wall during the DCR operation, the ideal position of nasal mucosal incision and bone resection can be obtained by referring to it. Our study shows that it is feasible to determine the level of the $\mathrm{CC}$ on the lateral nasal wall through the MTA, which is of great significance for accurate localization of the lacrimal sac and full opening of the lacrimal sac cavity in DCR surgery. There are few reports about the location of the $\mathrm{CC}$ relative to the MTA. The reason may be related to the difficulty in displaying the CC and the MTA on a coronal CT-DCG image at the same time, and the difficulty in directly measuring their distance. In this study, the distance from the axilla to the $\mathrm{CC}$ was obtained by calculating the difference 
between the distance from the SSF to the axilla and the distance from SSF to the CC. It is expected that there will be better measurement methods to further verify the positioning of the $\mathrm{CC}$ in the future.

Wormald et al. ${ }^{5}$ used CT-DCG to show that the fundus of the sac lies about $5 \mathrm{~mm}$ above the common canalicular opening. In our study, the average distance from the fundus of the sac to the CC was $3.41 \mathrm{~mm}$ $\pm 1.31 \mathrm{~mm}$. With the increase of the transverse diameter of the sac, the distance from the fundus of the sac to the CC gradually increases. The reason for the difference between the studies may be related to the ethnic origin of the research subjects ${ }^{6}$. In addition, since the previous studies seldom grouped according to the size of the sac, the composition ratio of the size of the sac may also be a factor leading to the differences.

In view of the importance of fully exposing the common canalicular opening in the sac during endoscopic DCR surgery, the MFP at the level of the CC must be removed during the operation. We measured the thickness of the MFP at the level of the CC, and found that the bone thickness was on average $3.50 \mathrm{~mm} \pm$ $1.24 \mathrm{~mm}$, which had no significant correlation with the size of the sac. This is very close to the results of previous studies in which the bone thickness of the MFP was $3-6 \mathrm{~mm}$ above the maxillary line ${ }^{6,9}$. In the process of powered endoscopic DCR, the height of mucosal incision was weakened by the drill grinding the thick bone of MFP as one progresses posteriorly ${ }^{6}$. Therefore, in order to fully open the lacrimal sac and reach the target height, the nasal mucosa incision should be designed with an additional $2-3 \mathrm{~mm}$ incision height.

In the DCR procedure, for patients in the large and medium lacrimal sac groups, there is a large space around the CC in the lacrimal sac. We believed that the design of the nasal mucosal incision need not aim at opening the fundus of the sac. And it should refer to the level of CC, increase the exposed height above the common canalicular opening by $2 \mathrm{~mm}$, and add an additional $2-3 \mathrm{~mm}$ to compensate for the loss of the incision height caused by the bone thickness of the MFP. According to the average distance from the axilla to the $\mathrm{CC}$ is $4 \mathrm{~mm}$ in our study, it is estimated that the position $8-9 \mathrm{~mm}$ above the axilla can be designed as the upper boundary of the endoscopic DCR nasal mucosa incision. This is consistent with Wormald et al. 's theory that the first mucosal incision should be made at 8 to $10 \mathrm{~mm}$ above the axilla ${ }^{12}$. Due to the abundance of nerves and vessels in the nasal roof, determining the location of mucosal incision can ensure the effective opening of the sac and CC, and reduce the risk of bleeding and injury of olfactory nerve fibers caused by excessively high incision design.

The particularity of the small lacrimal sac DCR operation lies in a thick sac wall, a narrow cavity, and less normal mucosal tissue around the CC, difficulty in exposing sac, and the small DCR ostium is easily blocked by granulation and cicatrix. Small lacrimal sac DCR surgery is difficult, and the success rate of surgery is not ideal ${ }^{13-14}$, which has always been a thorny problem in lacrimal drainage reconstruction surgery. The key to small lacrimal sac DCR surgery is to create a sufficient sac opening on lateral nasal wall with exposure of the $\mathrm{CC}$, increase the area of normal mucosa around the $\mathrm{CC}$, and reduce nasal mucosa damage and bone exposure over the location of the ostium ${ }^{13,15-16}$. Therefore, we convinced that the preoperative positioning of the lacrimal sac should be aimed at exposing the sac fundus. Thus can 
open the narrow diverticulum-like space between the sac fundus and the $\mathrm{CC}$, increase the area of the normal mucosa around the CC. Furthermore, in the small sac DCR operation, due to the small sac cavity and the small sac mucosal flap, the large mucosal incision made in conventional DCR surgery may not be suitable. The larger the mucosal excision, the more mucosal damage and the bare bone, which increases the risk of ostium granulomas proliferation and cicatricial closure ${ }^{17}$. Individual design of mucosal incision is of great significance to improve the success rate of small lacrimal sac DCR surgery.

In conclusion, our study shows that it is feasible to locate the sac through the relative position of the CC and the MTA. The distance of the SSF to the MTA was influenced by the lacrimal sac size. Accurately positioning the lacrimal sac and upper nasal mucosal incision is beneficial to fully open the lacrimal sac and expose the $\mathrm{CC}$. At the same time, it can avoids much more mucosal incisions than necessary during the operation, which can shorten the operation time, reduce the surgical trauma, and make the endoscopic DCR operation more safer, more efficient, and less invasive. The above is of great significance for improving the success rate of endoscopic DCR surgery. Since this study only discussed the availability of locating the lacrimal sac by CT-DCG, surgical data was limited. In the future, more locating information of lacrimal sac and nasal mucosa incision in endoscope DCR surgery should be collected to further supplement and verify the clinical application of the above results.

\section{Declarations}

Funding This work is supported by Science and Technology Fund of Tianjin Eye Hospital (YKYB2005).

Competing interests The authors declare no competing interests.

Correspondence and requests for materials should be addressed to L.H.Y.

\section{References}

1. Patella F, et al. The role of interventional radiology in the treatment of epiphora. Gland Surg 7, 103110. https://doi.org/10.21037/gs.2017.09.16 (2018).

2. Wormald PJ, Tsirbas A. Investigation and endoscopic treatment for functional and anatomical obstruction of the nasolacrimal duct system. Clin Otolaryngol Allied Sci 29, 352356.https://doi.org/10.1111/j.1365-2273.2004.00836.x (2004).

3. Wormald PJ. Powered endoscopic dacryocystorhinostomy. Otolaryngol Clin North Am 39, 539-549. https://doi.org/10.1016/j.otc.2006.01.009 (2006).

4. Konuk O, Kurtulmusoglu M, Knatova Z, Unal M. Unsuccessful lacrimal surgery: causative factors and results of surgical management in a tertiary referral center. Ophthalmologica 224, 361-366. https://doi.org/10.1159/000313818 (2010).

5. Wormald PJ, Kew J, Van Hasselt A. Intranasal anatomy of the nasolacrimal sac in endoscopic dacryocystorhinostomy. Otolaryngology Head and Neck Surgery 123, 307-310. https://doi.org/10.1067/mhn.2000.105416 (2000). 
6. Shams PN, Wormald PJ, Selva D. Anatomical landmarks of the lateral nasal wall: implications for endonasal lacrimal surgery. Curr Opin Ophthalmo/ 26, 408-415. https://doi.org/10.1097/ICU.0000000000000160 (2015).

7. Wormald PJ. Powered endoscopic dacryocystorhinostomy. Laryngoscope 112, 69-72. https://doi.org/10.1097/00005537-200201000-00013 (2002).

8. Orhan M, Saylam CY, Midilli R. Intranasal localization of the lacrimal sac. Arch Otolaryngol Head Neck Surg 135, 764-770. https://doi.org/10.1001/archoto.2009.94 (2009).

9. Woo KI, Maeng HS, Kim YD. Characteristics of intranasal structures for endonasal dacryocystorhinostomy in asians. Am J Ophthalmol 152, 491-498. https://doi.org/10.1016/j.ajo.2011.02.019 (2011).

10. Kakizaki $\mathrm{H}$,et al. Punctal and canalicular anatomy: implications for canalicular occlusion in severe dry eye. Am J Ophthalmo/ 153, 229-237. https://doi.org/10.1016/j.ajo.2011.07.010 (2012).

11. Welham RA, Wulc AE. Management of unsuccessful lacrimal surgery. Br J Ophthalmo/ 71, 152-157. https://doi.org/10.1136/bjo.71.2.152 (1987).

12. Roithmann R, Burman T, Wormald P. Endoscopic dacryocystorhinostomy. Braz J Otorhinolaryngo/ 78, 113-121. https://doi.org/10.5935/1808-8694.20120043 (2012).

13. Hammoudi DS, Tucker NA. Factors associated with outcome of endonasal dacryocystorhinostomy. Ophthalmic Plast Reconstr Surg 27, 266-269. https://doi.org/10.1097/IOP.0b013e31820c72b7 (2011).

14. Mannor GE, Millman AL. The prognostic value of preoperative dacryocystography in endoscopic intranasal dacryocystorhinostomy. Am J Ophthalmo/ 113, 134-137. https://doi.org/10.1016/s00029394(14)71524-7 (1992).

15. Kansu L, Aydin E, Avci S, et al. Comparison of surgical outcomes of endonasal dacryocystorhinostomy with or without mucosal flaps. Auris Nasus Larynx 36, 555-559. https://doi.org/10.1016/j.anl.2009.01.005 (2009).

16. Durvasula VS, Gatland DJ. Endoscopic dacrocystorhinostomy: long-term results and evolution of surgical technique. J Laryngol Oto/ 118, 628-632. https://doi.org/10.1258/0022215041917835 (2004).

17. Onerci $\mathrm{M}$, Orhan $\mathrm{M}$, Ogretmenoglu $\mathrm{O}$, Irkec $\mathrm{M}$. Long-term results and reasons for failure of intranasal endoscopic dacryocystorhinostomy. Acta Otolaryngo/ 120, 319-322. https://doi.org/10.1080/000164800750001170 (2000).

\section{Figures}




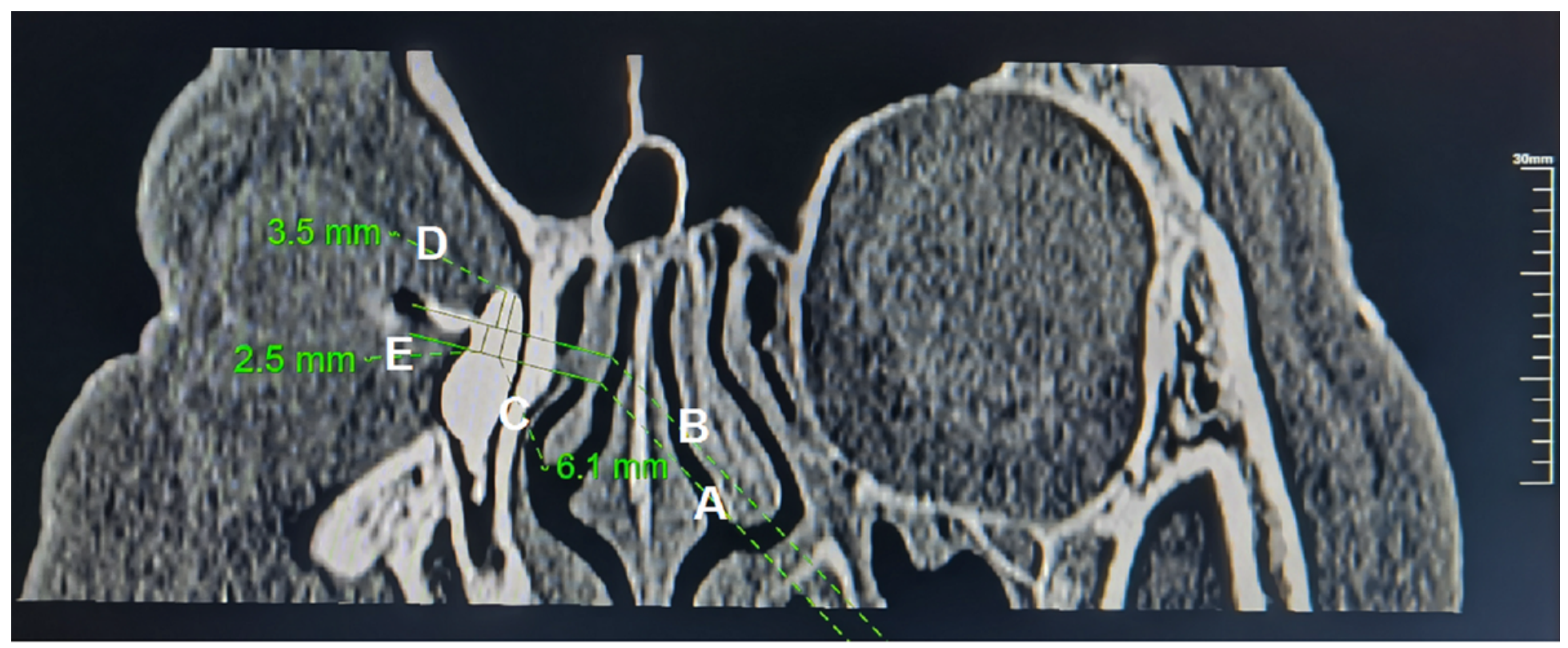

\section{Figure 1}

Measurements in the long axis of lacrimal sac on computed tomographic dacryocystography (CT-DCG) parasagittal images. A, a line drawn from the middle turbinate axilla (MTA) to the long axis of the sac at right angles. $B$, a line drawn through the common canaliculus (CC) to the long axis of the sac at right angles. $C$, the height of the sac above the MTA. D, the height of the sac above the CC. E, the distance from the MTA to the CC.
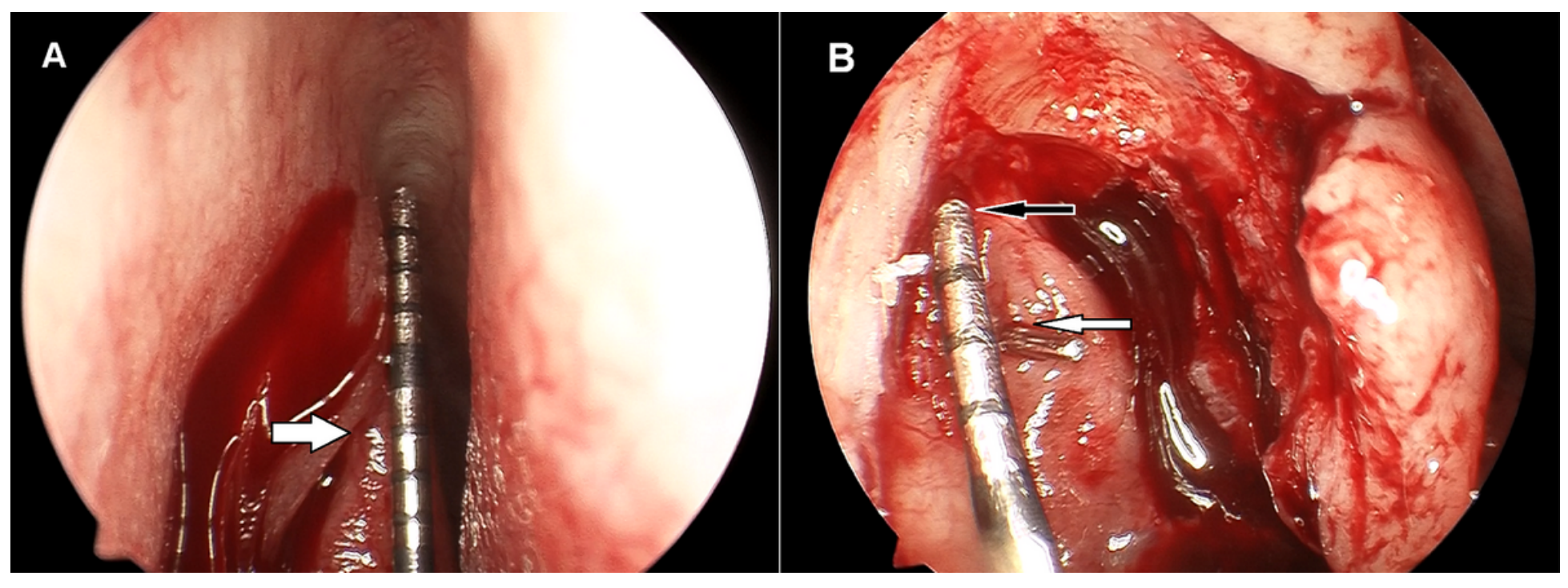

Figure 2

Intraoperative view of endoscopic dacryocystorhinostomy (endoscopic DCR). A, the sac location on the lateral nasal wall with graduated probe. The white arrow shows the middle turbinate axilla (MTA). B, The black arrow shows the exposed sac superior fundus (SSF) and the white arrow shows the opening of the common canaliculus (CC). Intraoperative measurements were performed. 

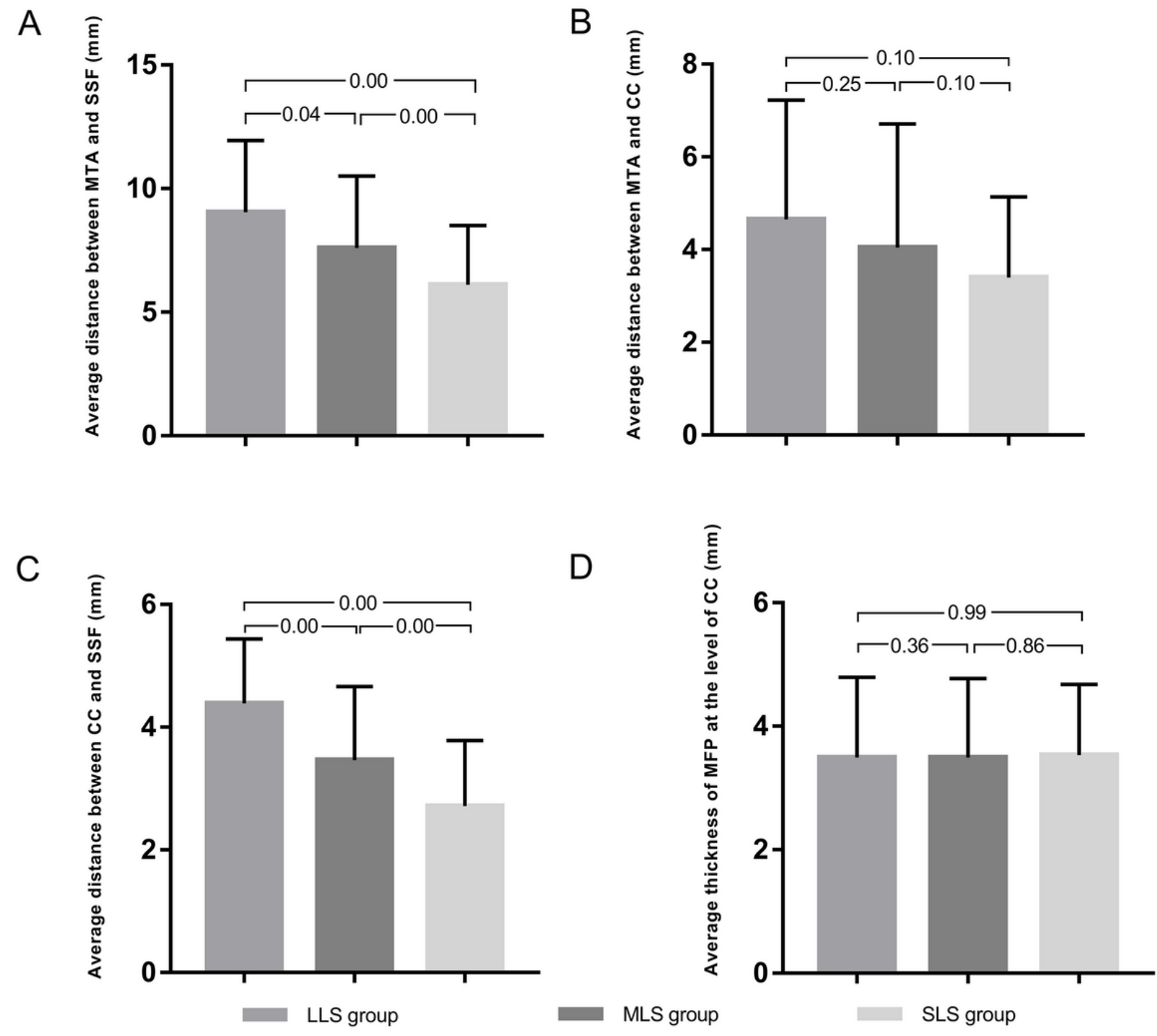

MLS group

SLS group

\section{Figure 3}

Comparison of measurements on computed tomographic dacryocystography (CT-DCG) images between groups. A, Average distance between the middle turbinate axilla (MTA) and the sac superior fundus (SSF); $B$, Average distance between the MTA and the common canaliculus (CC); C, Average distance between the CC and the SSF; D, Average thickness of maxillary frontal process (MFP) at the level of CC. Data are presented as mean \pm standard deviation. Numbers above the bars represent the $p$ values. Abbreviations: LLS, large lacrimal sac; MLS, medium lacrimal sac; SLS, small lacrimal sac. 